\title{
SUI POSSIBILI MODI DOSCILLAZIONE LIBERA \\ DEL LAGO DI BRACCIANO
}

\author{
Piftro Caloi
}

Il lago di Bracciano è per ampiezza il secondo dei laghi laziali; si trova a NW di Roma, ad una quarantina di km dalla Capitale, sulla destra della via Claudia, che tocca Bracciano, il maggiore dei centri abitati prospicienti il lago, dominati dalla superba mole del Castello Orsini.

Esso è cinto da colli selvosi, culminanti a Nord con il Monte di Rocca Romana, alto $602 \mathrm{~m}$. La cerchia montuosa degrada dolcemente lungo le rive meridionali, limitate da colline che si elevano di rado di un centinaio di metri sul lago, la cui superficie è a $164 \mathrm{~m}$ circa sul livello del mare. Oltre a Bracciano, danno sul lago altre cittadine: Anguillara Sabazia, sopra un caratteristico, ripido promontorio, Trevignano e Vicarello. È di una semplice, stupenda bellezza, sostanziata di mille diversi aspetti, che se non hanno la superba opulenza dei laghi lombardi e veneti, si compongono però in un complesso mirabile per serena eleganza e suggestivo equilibrio. A chi, procedendo sulla provinciale da Monterosi, balza all'occhio la visione della conca azzurra, inquieta di riflessi nella corona di verdi boschi e bianchi casolari, il cuore ha un tuffo di gioia. A chi, a sera di un limpido giorno di settembre, venendo da Contrada Polline (dove magari si è attardato nella sistemazione di un limnografo) procede sul lungolago di Trevignano, e intorno è l'incanto dolce del tramonto, e sul lago e nei boschi i colori si fondono alle ultime luci, sente nell'animo il fascino del luogo e dell'ora, incomparabile premio al lahorioso pomeriggio. A chi, nel mattino di una chiara giornata di febbraio, procede da Bracciano e s'inoltra nella riviera solatia da Vicarello a Trevignano, fra poggi olenti nel delicato trionfo di bianche rosee corolle di mandorli e peschi in fiore e la meraviglia di mimose - gialle nei fiori, verdi nelle fronde - e, superata Trevignano, prosegue sulla strada che circonda il piccolo, incantevole golfo - stupore di umili case cullate nel riflesso sull'onda... - l'assillo della mente, l'indomo slancio deli'animo, si placano in umiltà e in riconoscenza, 
in un senso d'amore per le cose, per le nostre care cose, per il nostro lavoro.

Sono questi, per il geofisico, $\mathrm{i}$ momenti in cui l'ansia della ricerra si sposa all'estasi dell'arte, in lampi d'acutissima sintesi, in cui si sublima il senso della vita.

Sul lago di Bracciano furono messi in funzione due limnografi: uno in località Valle dell'Inferno, dal settembre 1947 al maggio 1948, l'altro ad Anguillara Sabazia dal gennaio 1948 al febbraio 1949. Inoltre, per la durata di circa una settimana, tre limnografi funzionarono contemporaneamente a Vicarello, Bracciano ed Anguillara (fig. 1).

Lo spoglio delle osservazioni ottenute, alle quali è nostra intenzione aggiungerne altre, non è stato ancora compiuto; cosa che ci riserviamo di fare quanto prima.

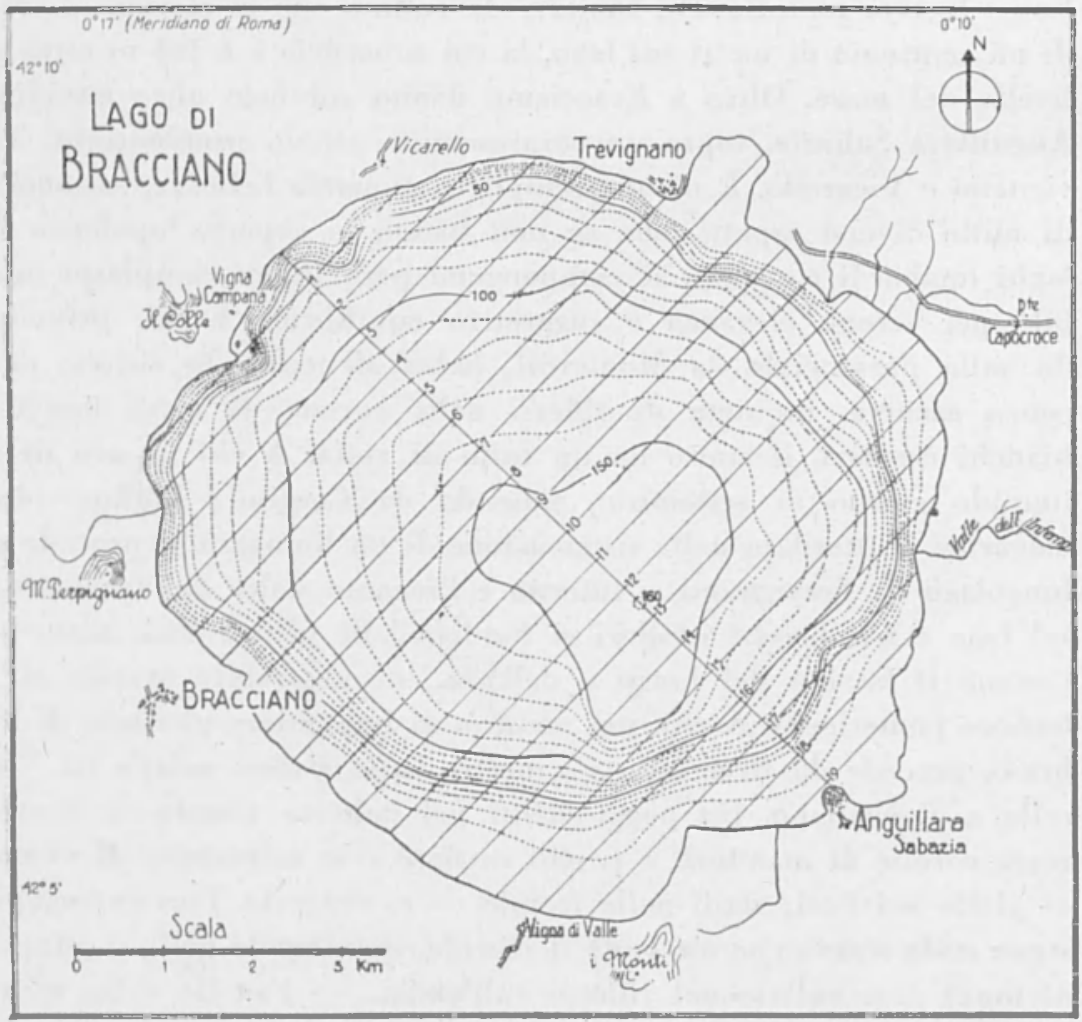

Fig. 1 
Scopo del presente lavoro è di mettere in chiaro, dal punto di vista analitico, a quali diverse conclusioni conduce il considerare il lago oscillante, nel suo insieme, come hacino a superficie circolare oppure come lacino a superficie rettangolare.

a) Il lago di Bracciano come bacino a forma circolare.

1. - Nel caso di un bacino a forma circolare, di profondità costante $h$, scegliendo l'origine delle coordinate al centro e trasformando in cordinate polari

$$
x=r \cos i, y=r \sin i,
$$

l'equazione delle oscillazioni libere dell'acqua racchiusa nel bacino stesso, diviene $\left(^{1}\right)$

$$
\frac{\partial^{2} \zeta}{\partial r^{2}}+\frac{1}{r} \frac{\partial^{2}}{\partial r}+\frac{1}{r^{2}} \partial y^{2} \zeta+k^{2} \zeta=0
$$

dove $\zeta$ è l'innalzamento della superficie lihera sopra il livello indisturlato ed è $k=\frac{2 \pi}{T c}$, essendo $T$ il periodo e $c=l \overline{g h}$ la velocita. Come al solito, si suppone trascurabile l'accelerazione verticale, cosi che uguale per tutte le particelle sulla stessa verticale risulta il moto orizzontale.

Si lıa allora per $\zeta$ (che dev'essere finito per $r=0$ ) l'espressione

$$
\zeta=A_{\mathrm{u}} J_{\mathrm{u}}(k r) \operatorname{sit}_{\operatorname{sil}}^{\cos }(\mathrm{s}) \cdot \cos \left(\frac{2 \pi}{i^{\prime}} t+\varepsilon\right),
$$

dove $s$ può avere uno dei valori $0,1,2,3, \ldots, A_{\mathrm{s}}$ ¿̀ una costante arlitraria $\mathrm{e} \frac{2 \pi}{T}=k c$. I valori ammissilili per $k$ sono determinati dalla condizione

$$
\frac{\partial}{\partial r}=0
$$

al limite $r=a$ ( $a$, raggio del hacino circolare), che si traduce nell'altra

$$
J_{\circ}^{\prime}(k a)=0 .
$$

Il caso $s=0$ corrisponde a moto simmetrico intorno al centroorigine: $\mathrm{i}$ nodi e $\mathrm{i}$ ventri avranno pertanto forma circolare.

Le più piccole radici dell'equazione

$$
J_{\mathrm{o}}^{\prime}(k a)=0,
$$


sono, come è noto (e come, comunque, può trarsi dalle tabelle sui valori delle funzioni di Bessel) ( $\left.{ }^{2}\right)$

$$
k a=3,832 ; 7,016 ; 10,173 ; \ldots . .
$$

Il moto asimmetrico (secondo un diametro) più interessante cor. risponde ad $s=1$ :

$$
\zeta=A J_{1}(k r) \cos \vartheta \cdot \cos \left(\frac{2 \pi}{T} t+e\right),
$$

dove $k$ è determinato dalla condizione

$$
J_{1}^{\prime}(k a)=0 .
$$

Il valori di ka clie soddisfano la [8] sono:

$$
l a=1,841 ; 5 ; 332 ; 8,536 ;
$$

La posizione del diametro nodale è, naturalmente, indeterminata.

La figura 2 rappresenta (secondo Lamb) le oscillazioni della superficie libera in corrispondenza delle sesse uninodale e linodale. Le
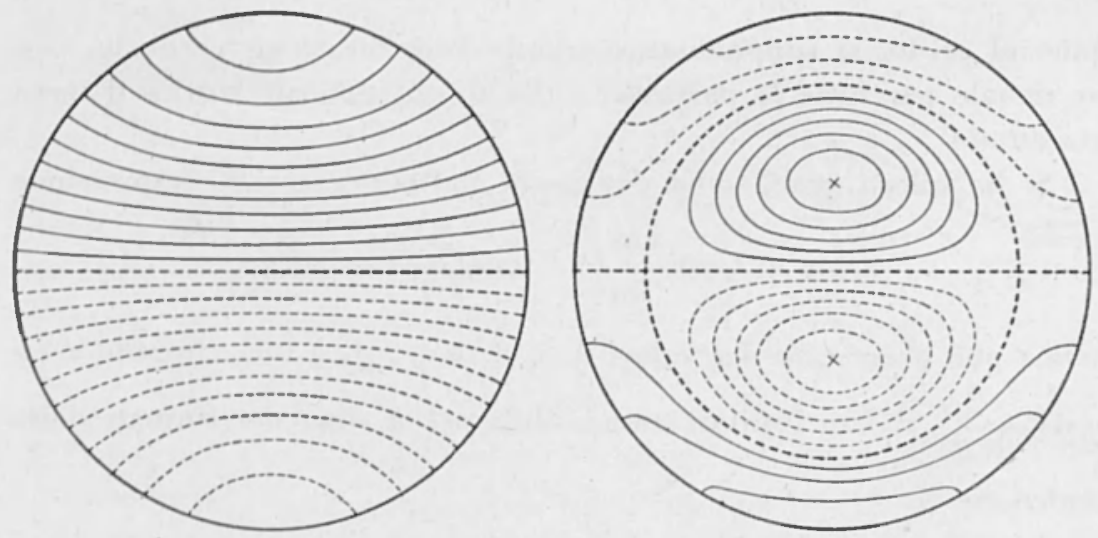

Fig. 2

linee di uguale spostamento tagliano il contorno ad angoli retti $\left({ }^{3}\right)$. Per l'oscillazione libera uninidole, il movimento ha molta analogia con quello uninodale di un canale chiuso ai due estremi. Nell'oscillazione binodale, uno dei binodi è una circonferenza, il cui raggio è dato dalla radice minore dell'equazione $J_{1}(k r)=o$ e vale $r=0.719 a$.

Consideriamo ora il caso di un bacino a profondità variabile.

Qualora la profonditi $h$ sia funzione soltanto della distanza $r$ dall'origine, l'equazione per le oscillazioni libere diventa ( $\left.{ }^{4}\right)$ 


$$
h \Delta_{2} \zeta+\frac{d h}{d r} \frac{\partial \zeta}{\partial \boldsymbol{r}}+\frac{4 \pi^{2}}{\boldsymbol{T}-\boldsymbol{g}} \zeta=0,
$$

dove $A_{2}$ è l'operatore di Laplace.

Se poi la profondità del bacino circolare decresce radialmente dal centro verso la periferia, secondo la legge

$$
h=h_{s}\left(1-\frac{r^{2}}{a^{2}}\right),
$$

introducendo coordinate polari e supponendo $\zeta$ variabile come $\cos s\}$ o $\sin s \vartheta$, la $[10]$ si può scrivere

$$
\left(1-\frac{r^{2}}{\boldsymbol{a}^{2}}\right)\left(\frac{\partial^{2} \zeta}{\partial \boldsymbol{r}^{2}}+\frac{1}{r} \frac{\partial \zeta}{\partial r}-\frac{s^{2}}{r^{2}} \zeta\right) \quad \frac{2}{\boldsymbol{a}^{\mathbf{2}}} r \frac{\partial \zeta}{\partial r}+\frac{4 \pi^{2}}{T^{2} g \boldsymbol{h}_{\mathbf{0}}} \zeta=0 .
$$

L'integrale della [12] può essere dato sotto forma di serie ascendente.

Si trova che dev'essere

$$
\frac{4 \pi^{2}}{T^{2} g h_{0}} a^{*}=(s+2 j)(s+2 j-2)-s^{3},
$$

dove $l$ è un intero (mağriore dell'unità nel caso simmetrico, maggiore di 0 per il caso asimmetrico).

Nol caso di oscillazione libera simmetrica $(s=0)$, ̀̀

$$
\frac{4 \pi^{2}}{T^{2}} \frac{a^{2}}{g h_{0}}=4 j(j-1 ; ;
$$

si avrà quindi, per le oscillazioni libere di nodalità crescente,

$$
\frac{2 \pi}{T} \frac{a}{\sqrt{g h_{0}}}=2,828 ; 4,899 ; 6,928 ;
$$

L'oscillazione uninodale $(j=2)$ ha un cerchio nodale di raggio 0.707 a. Per oscillazioni libere asimmetriche $(s=1)$, si ha dalla [13]

$$
\frac{4 \pi^{2}}{T^{2}} \cdot \frac{a^{2}}{g h_{0}}=4 j^{\prime}-2
$$

e quindi, per sesse uni-, bi-, trinodali,... avremo

$$
\frac{4 \pi}{T} \frac{a}{\sqrt{\mathrm{g} h_{0}}}=1,414 ; 3,742 ; 5.831 ;
$$

Per la sessa uninodale $(s=1, j=1$ ), la superficie libera è sempre piuna $(\overline{5})$.

Dal confronto con la [9] risulta che il periodo è $1 / .768$ di quello della corrispondente oscillazione in un bacino circolare di profondità uniforme ho e dello stesso raggio. 
2. - Applichiamo ora la teoria al lago di Bracciano.

Abhiamo:

$\begin{array}{lcc}\text { superficie totale } & \mathbf{k m}- & 57,5 \\ \text { raggio medio }(a) & \mathbf{k m} & 4,278 \\ \text { profondità media }(h) & \mathrm{m} & 86,1\end{array}$

Nel caso di moto asimmetrico, e profondità costante, pari alla media, i periodi delle tre prime sesse, come si deducono dalla [9], sono

$$
T_{0}=8^{\mathrm{m}}, 4 ; T_{1}=2^{\mathrm{m}}, 9 ; T_{\mathrm{v}}=1^{\mathrm{m}}, 8 .
$$

Sempre per profondità uniforme $(h=\mathbf{m} 86,1)$, nel caso di moto simmetrico la [6] ci consente di trarre i seguenti valori per i periodi delle prime tre sesse

$$
T_{0}=4^{\mathrm{m}}, 0 ; T_{1}=2^{\mathrm{m}}, 2 ; T_{2}=1^{\mathrm{m}}, 5 .
$$

Consideriamo ora il caso di profondità variabile radialmente.

Partendo dal centro del lago e procedendo radialmente nelle di-

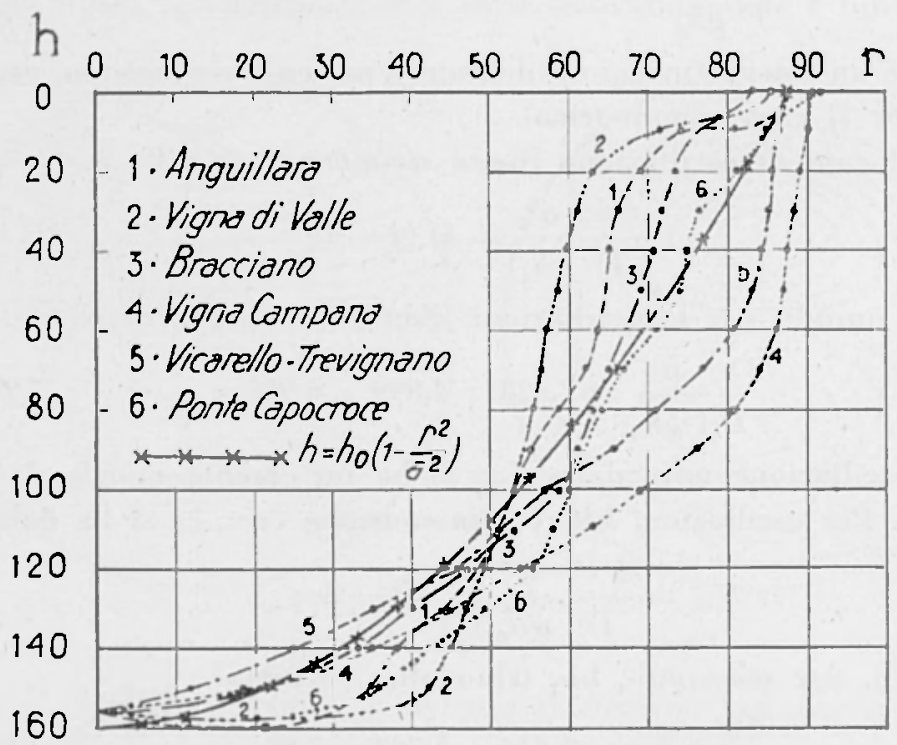

Fig. 3

rezioni di Anguillara, Vigna di Valle, Bracciano, Villa Campana, Vicarello-Trevignano e Ponte Capocroce l'andamento delle profondità come risulta dalla fig. 3 (dove le ascisse sono espresse in unità della scala, 1:50000).

La linea non numerata dà l'andamento secondo la [11], la quale, 
con buona approssimazione, può quindi ritenersi rappresentare l'andamento medio della profondità del lago.

Nel caso di oscillazioni libere asimmetriche, tenendo conto che ho $=160 \mathrm{~m}$, dalla [15] otteniamo per i periodi delle sesse uni-, bi- e trinodale i valori

$$
T_{0}=8^{\mathrm{m}}, 0 ; T_{1}=3^{-}, \hat{0} ; T_{\mathrm{g}}=1^{\mathrm{m}}, 9 .
$$

Nel caso di oscillazioni simmetriche, la [14] dà per l'uninodale il periodo

$$
T_{0}=4^{\mathrm{m}}, 0
$$

con il cerchio nodale di raggio $r=\mathrm{km} \mathrm{3,025.} \mathrm{Per} \mathrm{la} \mathrm{binodale} \mathrm{e} \mathrm{la}$ trinodale si hanno $\mathrm{i}$ valori

$$
T_{1}=2^{\mathrm{m}}, 3 ; T_{\mathrm{g}}=1^{\mathrm{m}}, 6 .
$$

b) Il. lago di Bracciano come bacino a forma pressoché rettangolare.

1. - In questa ipotesi, si può far uso di parecchi metodi. Ricorreremo a quello di Chrystal-Hidaka, riservandoci di pervenire alla soluzione con un procedimento particolare.

Ritenendo il lago un canale, a sezione variabile, e supponendo che l'ampiezza $\zeta$ delle sesse, ad una sezione $x$, sia data da

$$
\zeta=-\frac{d u}{d v},
$$

dove $v$ e l'area, misurata sulla superficie del lago, da una determinata origine, alla sezione $x$, l'equazione differenziale cui soddisfa $u$ è

$$
\frac{d^{2} u}{d v^{2}}+\frac{4 u^{2}}{g T^{2} \sigma(v)}=0
$$

İ questa l'equazione di Chrystal. In essa $T$ è il periodo della sessa, $g$ l'accelerazione di gravità e $\sigma(v)=b(v)$. $S(v)$, dove $b(v)$ è la larghezza e $S(v)$ l'area della sezione $v(o x)$. La funzione $\sigma(v)$ può essere valutata dai dati batimetrici del lago. La curva che rappresenta la relazione fra $v$ e $\sigma(v)$ è detta curva normale del lago.

Si deve aggiungere che le funzioni $u$ e $\sigma(v)$ si annullano entrambe alle due estremità del lago.

Tenendo conto di quest'ultima condizione e ponendo

$$
z=\frac{v}{A} \quad, \quad 0<z<1
$$


essendo $A$ l'area totale del lago, possiamo scrivere

$$
\sigma(z)=h z(1-z) \varphi(z),
$$

love $h$ è una costante. Potremo pertanto scrivere, in luogo della [16],

$$
\frac{d^{2} u}{d v^{2}}+\frac{\lambda u}{h z(1-z) \varphi(z)}=0
$$

avendo posto

$$
\lambda=\frac{4 \pi^{2} A^{2}}{g T^{2}} .
$$

llidaka propone di risolvere la [16], soggetta alle note condizioni ai limiti, determinando con il metodo di Ritz nel calcolo delle variazioni, il valore stazionario dell'integrale

$$
I(u)=\int_{0}^{1}\left\{\left(\frac{d z}{d z}\right)^{2}-\frac{2}{\sigma(z)} \cdot u^{2}\right\} d z .
$$

Ebbi già modo di applicare ripetutamente e di estendere tale metorlo (")).

Mi limiterò a ricordare che l'equazione in $\hat{i}$ cui giunge Hidaka, contiene, come coefficienti, comhinazioni degli integrali

$$
I_{\mathrm{n}}=\int_{0}^{1} \frac{z \cdot(1-z)^{\bar{\tau}} \cdot z^{\mathrm{n}}}{\sigma(z)} d z
$$

La determinazione di detti integrali, in genere, data la complessità delle curve normali, si ottiene per integrazione numerica. Poiché, nel caso attuale, la curva normale si presenta abhastanza semplice, ho ritenuto di calcolare grli integrali [20] per integrazione diretta, cercando di dare a $\sigma(z)$ un'opportuna rappresentazione analitica.

2. -- In un lavoro successivo, saranno riportati tutti gli clementi geometrici relativi al lago (sezioni, larghezze, valori di $v(x)$, ecc.). Qui riporto solo i valori della funzione $\sigma(z)$, relativi alle 20 sezioni in cui il lago stesso è stato diviso fra Vigna Campana e Anguillara.

Osscrviamo che, per la [17], la [20] diviene

$$
I_{\mathrm{n}}=\int_{0}^{1} \frac{z(1-z) z^{\mathrm{n}}}{h_{\varphi(}(z)} d z
$$




\begin{tabular}{|c|c|c|}
\hline$\stackrel{\varrho}{=}$ & 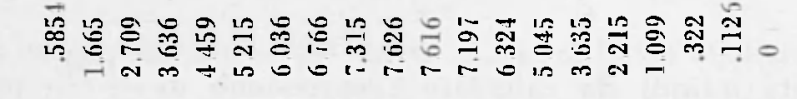 & \multirow{3}{*}{ 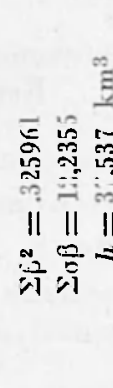 } \\
\hline$\ddot{\circ}$ & 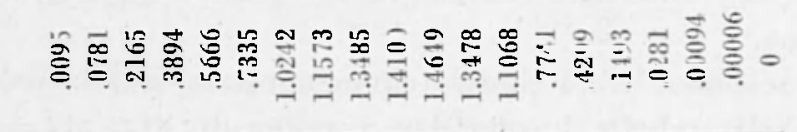 & \\
\hline$\ddot{n}$ & 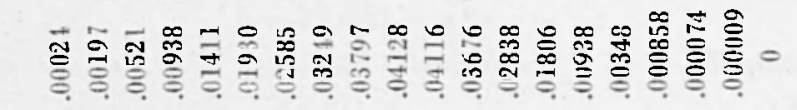 & \\
\hline$\infty$ & 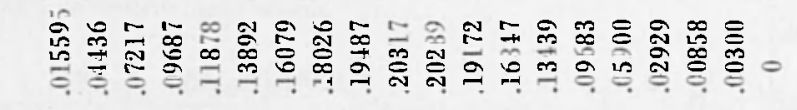 & \\
\hline i & 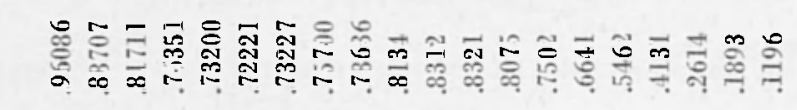 & \\
\hline 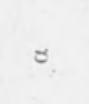 & 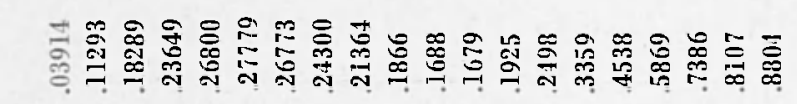 & \\
\hline $\begin{array}{l}\text { ڤै } \\
+\end{array}$ & 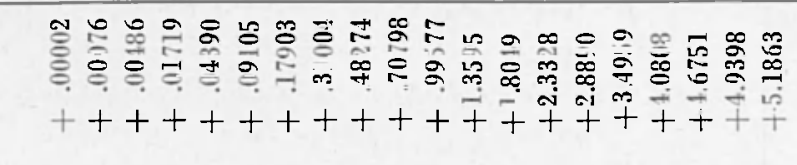 & \\
\hline $\begin{array}{l}\stackrel{1}{\infty} 8 \\
+\end{array}$ & 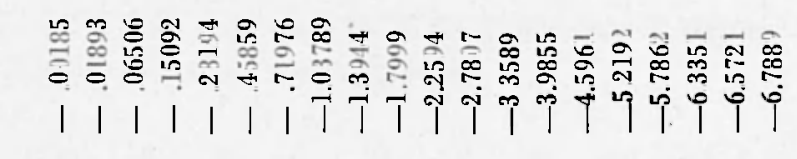 & 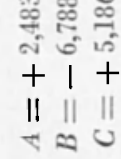 \\
\hline$\stackrel{n}{7}$ & 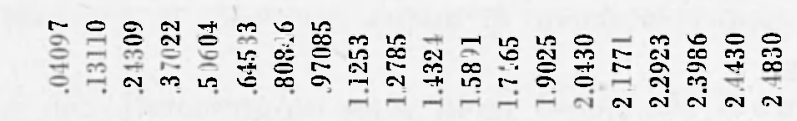 & \\
\hline$\frac{\bar{n}}{\frac{\pi}{i}}$ & 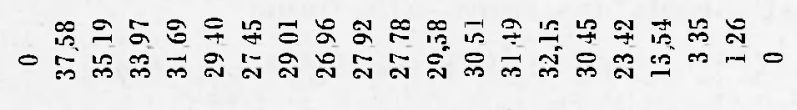 & \\
\hline$\frac{\pi}{1}$ & 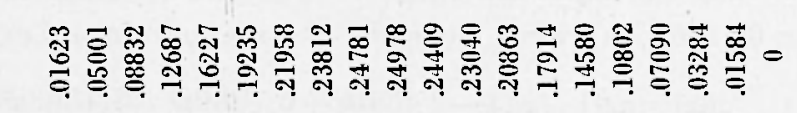 & \\
\hline$\widehat{E}$ & 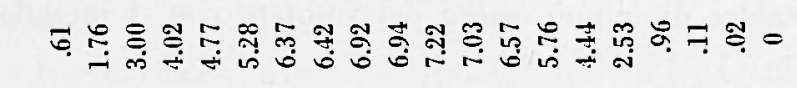 & \\
\hline$n$ & = & \\
\hline
\end{tabular}


Resta quindi da calcolare l'espressione di $\frac{\sigma(z)}{z(1-z)}$ per il lago di Bracciano.

La relazione fra $z$ e $\sigma(z)$ (curva normale) è data dai punti nella fig. 5. Nella tabella I sono dati i valori di $\pi(z) / z(1-z)$ per ogni sezione.

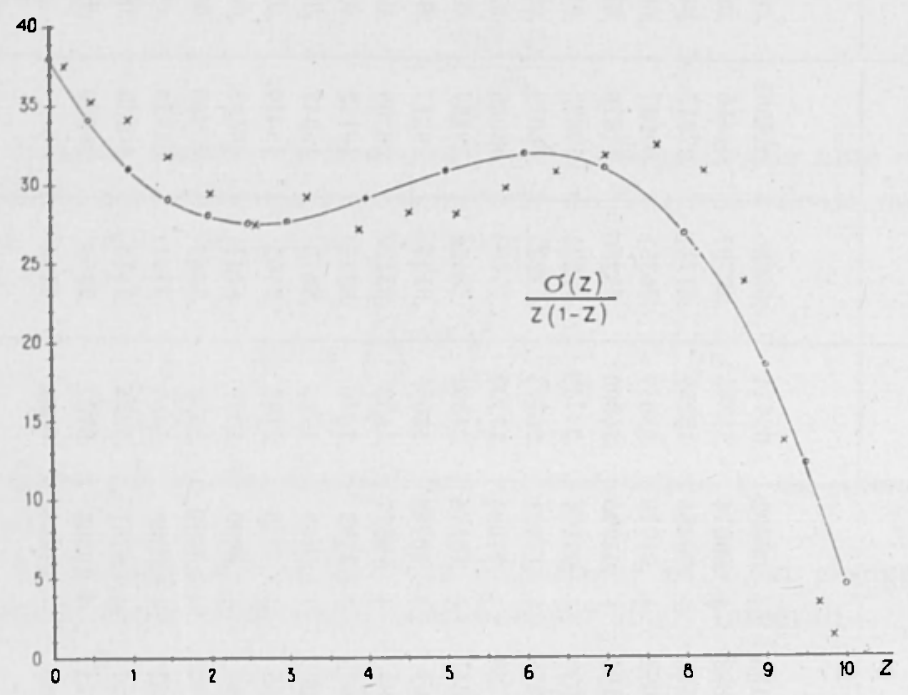

Fig. 4

La rappresentazione di questa grandezza in funzione di $z$ è data nella fig. 4.

Si trova che questi punti sono rappresentati, con sufficiente approssimazione, da una curva della forma

$$
h\left(1-2,4830 z+6,7889 z^{2}-5,1863 z^{3}\right),
$$

dove $h$ i una costante. Ia funzione $p(z)$ ha i valori estremi $q(o)=$ $1, \uparrow(1)=0.1196$. La curva normale assume pertanto l'espressione

$$
\sigma(z)=h z(1-z)\left(1-2,4830 z+6,7889 z^{2}-5,1863 z^{3}\right) .
$$

11 valore di $h$ può essere determinato con il metodo dei minimi quadrati.

Se poniamo

$$
\beta=z(1-z)\left(1-2,4830 z+6,7889 z^{2}-5,1863 z^{3}\right)
$$

ed indichiamo con $\sigma$ i valori osservati, dati nella tabella $I$, dobljiamo 
scegliere $h$ in modo che $\Sigma(\sigma--h \beta)^{2}$ sia un minimo. Si ha evidentemente

$$
h=\frac{\Sigma \sigma \beta}{\Sigma \beta^{2}}
$$

la sommatoria essendo estesa alle 20 sezioni. Ne consegue per $h$ il valore

$$
h=37,537 \mathrm{~km}^{3} \text {. }
$$

La fig. 5 dà la curva normale (osservata ++ e calcolata, linea continua).

Dalla $\left[20^{\prime}\right]$ avremo pertanto,

$$
I_{n}=\frac{1}{h} \int_{0}^{1} \frac{z(z-1) \cdot z^{\mathrm{n}} d z}{5,1863 z^{3}-6,7889 z^{2}+2,4830 z-1} \cdot\left[20^{\prime \prime}\right]
$$

Limitandoci al caso che corrisponde all'equazione di terzo grado in $A(")$

$$
\begin{aligned}
\left(I_{0} I_{2} I_{4}-I_{0} I^{2}{ }_{3}-I_{1}^{2} I_{4}+2 I_{1} I_{2} I_{3}-I_{2}{ }^{3}\right) \lambda^{3}-\left[\frac{3}{35}\left(I_{2} I_{0}-I_{2}\right)+\frac{1}{5}\left(-I_{0} I_{3}+I_{1} I_{2}+\right.\right. \\
\left.\left.+I_{1} I_{83}\right)+\frac{2}{15} I_{0} I_{4}+\frac{1}{3}-\left(-I_{1} I_{4}-I_{0}{ }^{2}+I_{2} I_{0}+I_{0} I_{4}-I_{2}\right)\right] \lambda^{2}+\left(\frac{1}{700} I_{0}-\right. \\
\left.\quad-\frac{3}{350} I_{2}+\frac{53}{2100} I_{2}-\frac{1}{30} I_{2}+\frac{1}{60} I_{2}\right) \lambda-\frac{1}{10500}=0 . \quad[21]
\end{aligned}
$$

risolvendo la $\left[20^{\prime \prime}\right]$ per $n=0,1,2,3,4$, otteniamo i seguenti valori per $I_{0} \ldots I_{+}$(scegliendo come unità il $\mathbf{M m}$ ):

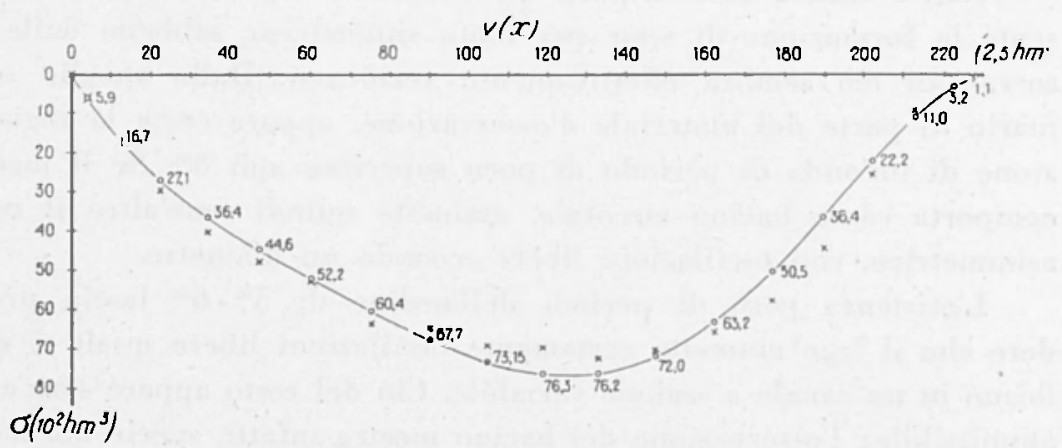

Fig. 5

$I_{0}=6,012693 ; I_{1}=3,118568 ; I_{2}=1,956829 ; I_{3}=1,371697 ; I_{4}=1,031885$.

Sostituiti in $(21)$, si ottiene un'equazione di $3^{0}$ grado in $\%$ che risolta conduce ai valori

$$
\lambda_{0}=055016 ; \lambda_{1}=\cdot 141798 ; \lambda_{2}=\cdot 299756 \text {. }
$$


Dalla [18] - essendo $A=0.57552 \mathrm{Mm}^{2}-$ conseguono per le sesse uni-, bi- e trinodali i valori

$$
T_{0}=8^{\mathrm{m}}, 2 ; T_{1}=5^{\mathrm{m}} \cdot 1 ; T_{2}=3^{\mathrm{m}}, 5 .
$$

c) Riassumiamo i risultati ottenuti nelle due ipotesi che considerano il lago di Bracciano assimilabile ad un bacino circolare o ad un cánale a sezione variabile. Essi sono compendiati nella tabella II.

T'ABELla II

l'ossibili modi d'oscillazione libera del lago di Bracciano

\begin{tabular}{|c|c|c|c|c|c|}
\hline \multirow{3}{*}{ 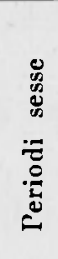 } & \multicolumn{4}{|c|}{ Bacino circolare } & \multirow{3}{*}{$\begin{array}{c}\text { Canale a } \\
\text { sezione } \\
\text { variabile }\end{array}$} \\
\hline & \multicolumn{2}{|c|}{ Moto simmetrico } & \multicolumn{2}{|c|}{ Moto asimmetrico } & \\
\hline & $\begin{array}{l}\text { Profondità } \\
\text { uniforme }\end{array}$ & $\begin{array}{c}\text { Profondità } \\
\text { viriabile } \\
h=h_{0}\left(1-\frac{r^{2}}{a_{2}}\right)\end{array}$ & $\begin{array}{c}\text { Profondità } \\
\text { uniforme }\end{array}$ & $\begin{array}{c}\begin{array}{c}\text { Profondità } \\
\text { variabile }\end{array} \\
h=h_{1}\left(1-\frac{r^{2}}{a_{2}}\right)\end{array}$ & \\
\hline $\mathrm{T}_{0}$ & $4, \mathrm{~m} 0$ & $4, \mathrm{~m} 0$ & $8, \mathrm{~m} 4$ & $8, \mathrm{~m} 0$ & $8, \mathrm{~m} 2$ \\
\hline $\mathrm{T}_{\mathrm{t}}$ & $2 . m 2$ & $2, \mathrm{~m} 3$ & $2 \mathrm{~m} 9$ & $3, \mathrm{~m} 0$ & $5, \mathrm{mI} \quad 1$ \\
\hline $\mathbf{T}_{2}$ & $1, \mathrm{~m} 5$ & $1 .{ }^{\mathrm{m}} 6$ & $1, \mathrm{~m} 8$ & $1, \mathrm{~m} 9$ & $3, \mathrm{~m} 5$ \\
\hline
\end{tabular}

Non è ancora dato di poter stabilire se il lago di Bracciano consente la formazione di sesse per moto simmetrico, sebbene dalle osservazioni ciò sembra effettivamente verificarsi. Dallo spoglio sommario di parte del materiale d'osservazione, appare certa la registrazione di un'onda di periodo di poco superiore agli $8^{\mathrm{m}}$. Se il lago si comporta come bacino circolare, ammette quindi senz'altro il moto asimmetrico, con oscillazioni libere secondo un diametro.

L'esistenza però di periodi dell'ordine di $5^{\mathrm{m}}-6^{\mathrm{m}}$ lascia prevedere che il lago ammette certamente oscillazioni libere quali si verificano in un canale a sezione variabile. Ciò del resto appare senz'altro ammissibile: l'osservazione del hacino mostra infatti, specie nel tratto includente Bracciano, tratti di costa pressoché rettilinei.

Comunque, ci riserviamo di chiarire in un prossimo lavoro tutti $i$ possibili modi d'oscillazione del lago di Bracciano: il largo materiale d'osservazione già raccolto, e quello che ci proponiamo di ottenere ancora, faciliterà certamente il nostro compito.

Roma - Istituto Nazionale di Geofisica - Gennaio 1950. 


\section{RIASSUNTO}

In vista di un'accurata interpretazione delle registrazioni delle sesse del lago di Bracciano, viene studiato analiticamente il comportamento del lago, nelle sue possibili oscillazioni libere. Considerando il bacino come circolare, si determinano $i$ valori dei periodi delle sue oscillazioni libere simmetriche e asimmetriche, nei due casi di profondità uniforme e di profondità variabile. Infine, si considera l'eventualità di un comportamento conforme a quello di un canale a sezione variabile. Sia il caso asimmetrico, sia l'ultima eventualità conducono ad un periodo dell'uninodale coincidente con quello dato dall'osservazione. In un prossimo lavoro, verrà riportato l'esame delle registrazioni ottenute e il confronto con $i$ dati forniti dalla teoria.

\section{BIBLIOGRAFIA}

(1) Lamb H.: Hydrodynamics. Cambridge (1932), pag. 284.

(2) Jahnke-Emde: Funktionentafeln. Teubner in Leipzig (1933), pag. 166.

(3) Lamb H.: l. c., pag. 287.

(4) Lamb H.: l. c., pag. 291.

(5) LaMb H.: $l$. c., pag. 293.

(6) Caloi P.: Le sesse del lago di Garda; I. Annali di Geofisica, I, 1 (1948); II. Annali di Geofisica, I, 2 (1948). 